\title{
Tendon Reflex Decreased, CTCAE
}

National Cancer Institute

\section{Source}

National Cancer Institute. Tendon Reflex Decreased, CT CAE. NCI Thesaurus. Code C143234.

A disorder characterized by less than normal deep tendon reflexes. 